\title{
Malnutrition inequalities in Ecuador: differences by wealth, education level and ethnicity
}

\author{
María J Ramírez-Luzuriaga ${ }^{1, *,} \uparrow$ (D), Philippe Belmont ${ }^{2}$, William F Waters ${ }^{2}$ and \\ Wilma B Freire ${ }^{2}$ \\ 'Nutrition and Health Science Program, Laney Graduate School, Emory University, Atlanta, \\ GA, USA: ${ }^{2}$ Institute for Research in Health and Nutrition, San Francisco de Quito University, \\ Quito, Ecuador
}

Submitted 6 January 2019: Final revision received 1 June 2019: Accepted 18 June 2019: First published online 27 September 2019

\begin{abstract}
Objective: To describe and quantify the magnitude and distribution of stunting, wasting, anaemia, overweight and obesity by wealth, level of education and ethnicity in Ecuador.

Design: We used nationally representative data from the 2012 Ecuadorian National Health and Nutrition Survey. We used the Multidimensional Poverty Index (MPI) as a proxy of wealth. The MPI identifies deprivations across three dimensions (health, education and standard of living). We defined education by years of schooling and ethnicity as a social construct, based on shared social, cultural and historical experiences, using Ecuadorian census categories.

Setting: Urban and rural Ecuador, including the Amazon rainforest and the Galapagos Islands.

Participants: Children aged $<5$ years ( $n$ 8580), adolescent women aged 11-19 years ( $n$ 4043) and adult women aged $20-49$ years ( $n 15203$ ).

Results: Among children $<5$ years, stunting and anaemia disproportionately affected low-wealth, low-education and indigenous groups. Among adolescent and adult women, higher rates of stunting, overweight and obesity were observed in the low-education and low-wealth groups. Stunting and short stature rates were higher in indigenous women, whereas overweight and obesity rates were higher in Afro-Ecuadorian women.

Conclusions: Malnutrition differs significantly across sociodemographic groups, disproportionately affecting those in the low wealth tertile and ethnic minorities. Rates of stunting remain high compared with other countries in the region with similar economic development. The effective implementation of double-duty actions with the potential to impact both sides of the double burden is urgently required.
\end{abstract}

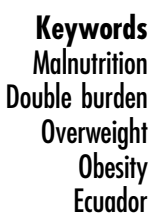

Maternal and child malnutrition in middle-income countries such as Ecuador has traditionally been the focus of nutrition agendas and encompasses both undernutrition and a growing and mostly unrecognized problem of overweight and obesity.

The prevalence of stunting in children $<5$ years of age has declined from $40 \cdot 2 \%$ in 1986 to $25.3 \%$ in 2012 , but remains high in some regions and sub-populations ${ }^{(1,2)}$. While undernutrition in the form of stunting and micronutrient deficiencies have been observed in Ecuador for at least two decades ${ }^{(2)}$, the emerging phenomenon of excess weight is still not widely recognized. In 1986, the combined prevalence of overweight and obesity in children $<5$ years was $4 \cdot 2 \%$, and by 2013 this proportion had doubled to $8.6 \%{ }^{(1)}$. Among women, overweight and obesity rates increase sharply from puberty to adult age, affecting $29.2 \%$ of adolescent and $63.8 \%$ of adult women $^{(1)}$. Furthermore, undernutrition coexists with

†Correspondence address: Mailstop 1518-002-7BB, 1518 Clifton Road, NE-CNR Room 7000-E, Atlanta, GA 30322, USA.

Disclaimer: This paper was published as part of a Supplement in the Journal of Public Health Nutrition, publication of which was supported partially by SLAN CAPÍTULO MEXICO A.C. The papers included in this supplement were invited by the Guest Editor and have undergone the standard journal formal review process. They may be cited. 
overweight and obesity ${ }^{(3)}$. This double burden of nutrition has been associated with rapid urbanization, economic growth and greater penetration of the retail food industry, which have resulted in diets based on energy-dense and nutrient-poor foods ${ }^{(4)}$. In countries like Ecuador, the double burden of malnutrition exacerbates as undernutrition problems have not been adequately addressed.

According to the World Bank, Ecuador is classified as an upper-middle-income country. The population living below the poverty line decreased from $64.4 \%$ in 2000 to $22.5 \%$ in $2014^{(5)}$. While rapid social and economic development has made modest inroads in the face of persistent poverty and inequality, the country remains highly unequal, which has disproportionately affected the nutritional status of the population ${ }^{(6,7)}$.

Given Ecuador's socio-economic disparities, the extent to which the distribution of various forms of malnutrition varies in different segments of the population has not been adequately explored. The aim of the present study was to assess the main nutrition problems affecting children and women of reproductive age in Ecuador stratified by wealth, level of education and ethnicity.

\section{Methods}

The present study is part of a supplement assessing malnutrition inequalities in ten countries in Latin America (Argentina, Bolivia, Brazil, Colombia, Chile, Ecuador, Guatemala, Mexico, Peru and Uruguay). A standard set of criteria was applied to allow between-country comparability. At a minimum, the population for analysis was required to be representative at the national level and include anthropometric and socio-economic measures in children aged $<5$ years, adolescent women aged 11-19 years and adult women aged 20-49 years. Nutritional measures were defined according to WHO standards, and the operationalization of education level was the same in all countries. Definitions for wealth and ethnicity varied in each country, depending on data availability and demographic characteristics.

In the present analysis, we followed the criteria previously described. We used nationally representative data from the 2012 Ecuadorian National Health and Nutrition Survey (ENSANUT-ECU). The sample is representative at the national and sub-regional levels: urban and rural Sierra (highland), urban and rural Coast, Amazon, Galápagos, and the cities of Quito and Guayaquil. The sample included a total of 57727 individuals and 19803 households ${ }^{(8)}$. Detailed information about the sampling methodology has been published elsewhere ${ }^{(1,8)}$.

Trained field workers collected information on sociodemographic characteristics and performed anthropometric measurements for all participants in the selected households using standardized procedures, protocols and equipment ${ }^{(9)}$. Age was confirmed by observing each individual's national identity card. Height was measured in participants $>2$ years old using portable stadiometers and length was measured in children aged $<2$ years using infantometers, to the nearest $0 \cdot 1 \mathrm{~cm}$. Electronic scales were used to estimate weight in children and adults to the nearest $0 \cdot 1 \mathrm{~kg}$. Anthropometric data were collected twice for each parameter to ensure reliability with an interval of 5 to $10 \mathrm{~min}$. Additionally, supervisors remeasured participants in every tenth household and interviewers were retrained after $11 \mathrm{~d}$ of fieldwork.

From a sub-sample of participants, trained phlebotomists drew venous blood samples after an $8 \mathrm{~h}$ fast, using standard methods, into trace-element-free vials. The blood was centrifuged in situ at $3500 \mathrm{rpm}$ for $10 \mathrm{~min}$, aliquoted and refrigerated. Serum was stored in cryotubes covered with aluminium foil to preserve them from light and kept in liquid nitrogen to be transported to the ENSANUT-ECU reference laboratory at Quito, Ecuador. Hb was measured using sodium lauryl sulfate spectrophotometry ${ }^{(10)}$.

\section{Study participants}

We included children aged $<5$ years and women of reproductive age (adolescents aged 11-19 years and adults aged 20-49 years). It is well established that these segments of the population are at higher risk of malnutrition due to social and biological factors. The final sample included 8580 children $<5$ years, 4043 adolescent women (11-19 years) and 15203 adult women (20-49 years) with complete anthropometric data. For biochemical assessments, the final sample included 2046 children < 5 years, 2084 adolescent women (11-19 years) and 7396 adult women (20-49 years) with complete $\mathrm{Hb}$ data. We excluded from the analysis pregnant women and individuals with missing socio-economic, anthropometric, demographic and $\mathrm{Hb}$ information.

\section{Malnutrition assessment}

To assess nutritional status in children of pre-school age and adolescent women, we calculated $Z$-scores using the WHO growth reference standards ${ }^{(11,12)}$. Stunting in children $<5$ years and adolescent women (11-19 years) was classified as length- or height-for-age $Z$-score $(\mathrm{HAZ})<-2$. For adult women (20-49 years), short stature was used as a proxy of stunting, classified as height $<1.49 \mathrm{~m}^{(13)}$.

For children $<5$ years, wasting was defined as weightfor-height $Z$-score $(\mathrm{WHZ})<-2$. For adolescent women (11-19 years) underweight was defined as BMI-for-age $Z$-score $(\mathrm{BMIZ})<-2$. For adult women $(20-49$ years) underweight was defined as BMI $<18.5 \mathrm{~kg} / \mathrm{m}^{2(14)}$.

Anaemia was defined using WHO cut-off points ${ }^{(15)}$. $\mathrm{Hb}$ values were adjusted for altitude using the method proposed by Nestel and adjusted by the Centers for Disease Control and Prevention's Pediatric Nutrition Surveillance System ${ }^{(16)}$.

For children $<5$ years, overweight was defined as BMIZ between $>2$ and $\leq+3$, and obesity as BMIZ $>+3$. For adolescent women (11-19 years), overweight was determined by BMIZ between $>+1$ and $\leq+2$, and obesity as BMIZ $>+2$. In adult women (20-49 years), excess weight was defined according to WHO standards using 
the BMI cut-off points of $\geq 25$ and $<30 \mathrm{~kg} / \mathrm{m}^{2}$ for overweight and $\geq 30 \mathrm{~kg} / \mathrm{m}^{2}$ for obesity ${ }^{(14)}$. Weight and height outliers for individuals aged $<19$ years were defined using the WHO SD boundaries; for adults, outliers were set at $5 \mathrm{SD}$ above or below the reference mean. Outliers were identified and excluded from the analyses.

\section{Wealth, education and etbnicity measures}

We used the global Multidimensional Poverty Index (MPI) to assess poverty at the individual level using ten indicators to identify deprivations across three dimensions: health, education and standard of living ${ }^{(17,18)}$. Poverty was defined if a person is deprived in at least one-third of the ten weighted indicators. Within each domain, every indicator was weighted equally. We divided the MPI into tertiles and used this measure as a proxy of wealth.

For children < 5 years and adolescent women (11-19 years), level of education was defined by the mother's years of schooling and categorized as low (0-6 years; primary school or less), medium (7-12 years; high school) or high (more than 12 years; more than high school). For adult women (20-49 years), level of education was defined by the women's years of schooling using the same categories previously described.

We defined ethnicity as a social construct, based on shared social, cultural and historical experiences, using categories included in the most recent (2010) census ${ }^{(6,19)}$. The three groups analysed were indigenous, AfroEcuadorian and mestizo.

\section{Statistical analyses}

Means and percentages with 95\% CI were estimated for sociodemographic and nutritional measures in children $<5$ years, adolescent women (11-19 years) and adult women (20-49 years) stratified by tertiles of wealth, education level and ethnicity. Differences in means and proportions between subgroup categories were tested using the test for linear combinations (lincom command in the statistical software package Stata version 12.0). A $P$ value of $<0.05$ was used to assess statistical significance. All statistical procedures were performed with Stata version 12.0 considering the complex design of the survey sample (SVY module).

\section{Results}

Among adult women, low level of education ranged from $16.5 \%$ in the high wealth tertile to more than half $(57.3 \%)$ in the low wealth tertile. Households in the medium and high wealth tertiles had greater access to public services such as a sanitary sewer system and public water networks. Access to electricity was universal with no differences observed across wealth tertiles (Table 1).

\section{Children aged $<5$ years}

Only stunting and anaemia differed significantly by sociodemographic characteristics. Rates of stunting and anaemia were significantly higher in the low tertile, relative to the medium and high wealth tertiles. Additionally, stunting and anaemia disproportionately affected indigenous children, relative to Afro-Ecuadorian and mestizo (Table 2 and Fig. 1).

No significant differences in overweight and obesity prevalences were observed across sociodemographic characteristics for children aged $<5$ years.

\section{Adolescent women aged 11-19 years}

Regarding undernutrition, $20.6 \%$ of adolescent women were stunted, $9.3 \%$ had anaemia and $1.0 \%$ suffered from wasting. The prevalence of stunting was significantly higher among adolescent women in the low education and wealth tertiles relative to adolescent women in the medium and high education and wealth tertiles (Table 2). Regarding ethnicity, half of indigenous adolescent women were stunted $(50 \cdot 1 \%)$; this proportion was significantly higher than that of Afro-Ecuadorian (11.2\%) and mestizo (18.8\%) adolescent women.

Regarding excess weight, Afro-Ecuadorian adolescent women were more affected by overweight and obesity relative to indigenous and mestizo women. No significant differences in rates of overweight and obesity among adolescent women were observed across tertiles of wealth and education.

\section{Adult women aged 20-49 years}

Undernutrition in the form of anaemia and short stature were prevalent, while the proportion of thinness was very small. Regarding ethnicity, the prevalence of short stature disproportionately affected indigenous women (51.8\%) compared with their Afro-Ecuadorian (11.8\%) and mestizo (26.7\%) counterparts.

The prevalence of overweight and obesity was significantly lower in women in the high wealth tertile $(60.0 \%)$ than in women in the medium and low tertiles ( 66.1 and $66.3 \%$, respectively). Furthermore, women with low level of education had a higher combined prevalence of overweight and obesity (69.8\%) compared with women with high level of education (58.6\%). Significant ethnic differences in rates of overweight and obesity were observed among adult women. Indigenous women had a higher proportion of overweight (41.3\%) than mestizo (38.9\%) and Afro-Ecuadorian women $(31.8 \%)$. However, the rate of obesity among Afro-Ecuadorian women was substantially higher $(34.9 \%)$ than that among mestizo (25.4\%) and indigenous women (13.3\%; Table 2). 
Table 1 Sample characteristics, overall and by tertile of wealth, in Ecuador (data are from the Ecuadorian National Health and Nutrition Survey 2012)

\begin{tabular}{|c|c|c|c|c|c|c|c|c|}
\hline & & & \multicolumn{6}{|c|}{ Wealth } \\
\hline & \multicolumn{2}{|c|}{ All } & \multicolumn{2}{|c|}{ Low tertile } & \multicolumn{2}{|c|}{ Medium tertile } & \multicolumn{2}{|c|}{ High tertile } \\
\hline & Mean or \% & $95 \% \mathrm{Cl}$ & Mean or $\%$ & $95 \% \mathrm{Cl}$ & Mean or $\%$ & $95 \% \mathrm{Cl}$ & Mean or \% & $95 \% \mathrm{Cl}$ \\
\hline \multicolumn{9}{|l|}{ Children aged $<5$ years $(n 8580)$} \\
\hline Mean age (years) & 2.49 & $2 \cdot 45,2 \cdot 54$ & $2 \cdot 51$ & $2.44,2.59$ & $2 \cdot 51$ & $2 \cdot 43,2 \cdot 60$ & $2 \cdot 46$ & $2 \cdot 38,2.53$ \\
\hline Male sex (\%) & $51 \cdot 0$ & $49 \cdot 5,52 \cdot 5$ & 49.5 & $47 \cdot 0,52 \cdot 0$ & $51 \cdot 2$ & $48 \cdot 0,54 \cdot 4$ & $52 \cdot 2$ & $49 \cdot 6,54.9$ \\
\hline \multicolumn{9}{|l|}{ Adolescent women aged $11-19$ years ( $n$ 4043) } \\
\hline $\begin{array}{l}\text { Mean age (years) } \\
\text { Adult }\end{array}$ & $15 \cdot 3$ & $15 \cdot 2,15 \cdot 4$ & 14.9 & $14 \cdot 8,15 \cdot 1$ & $15 \cdot 1$ & $14 \cdot 9,15 \cdot 3$ & $16 \cdot 1$ & $15 \cdot 9,16 \cdot 3$ \\
\hline Mean age (years) & 33.7 & $33.4,33.9$ & $34 \cdot 1$ & $33 \cdot 7,34 \cdot 6$ & $34 \cdot 1$ & $33 \cdot 7,34.5$ & 32.9 & $32.5,33.4$ \\
\hline \multicolumn{9}{|l|}{ Education level (\%) } \\
\hline Low (0-6 years) & 33.4 & $31 \cdot 7,35 \cdot 1$ & $57 \cdot 3$ & $55 \cdot 1,59 \cdot 5$ & $33 \cdot 1$ & $30 \cdot 9,35 \cdot 4$ & $16 \cdot 5$ & $14 \cdot 9,18 \cdot 2$ \\
\hline Medium (7-12 years) & $22 \cdot 4$ & $21 \cdot 3,23 \cdot 5$ & 23.2 & $21 \cdot 4,25 \cdot 2$ & $25 \cdot 2$ & $23 \cdot 2,27 \cdot 2$ & $19 \cdot 6$ & $17 \cdot 8,21 \cdot 5$ \\
\hline High ( $>12$ years) & $44 \cdot 2$ & $42 \cdot 3,46 \cdot 1$ & 19.4 & $17 \cdot 6,21.4$ & $41 \cdot \overline{7}$ & $39 \cdot 2,44 \cdot 3$ & 63.9 & $61 \cdot 5,66 \cdot 3$ \\
\hline \multicolumn{9}{|l|}{ Households ( $n 19006)$} \\
\hline \multicolumn{9}{|l|}{ Ethnicity (\%) } \\
\hline Indigenous & $5 \cdot 6$ & $4.9,6.5$ & 9.9 & $8 \cdot 3,11 \cdot 7$ & 4.6 & $3 \cdot 8,5 \cdot 7$ & 3.4 & $2 \cdot 7,4 \cdot 3$ \\
\hline Afro-Ecuadorian & 4.7 & $4 \cdot 1,5 \cdot 5$ & 4.9 & $3 \cdot 9,6 \cdot 2$ & $5 \cdot 8$ & $4 \cdot 6,7 \cdot 3$ & $3 \cdot 7$ & $3 \cdot 0,4 \cdot 6$ \\
\hline Mestizo & 89.6 & $88 \cdot 6,90 \cdot 6$ & $85 \cdot 2$ & $83 \cdot 1,87 \cdot 1$ & 89.5 & $87 \cdot 9,91 \cdot 0$ & $92 \cdot 8$ & $91 \cdot 7,93 \cdot 8$ \\
\hline \multicolumn{9}{|l|}{ Household characteristics and goods (\%) } \\
\hline Bare-earth floor & $3 \cdot 8$ & $3 \cdot 2,4.4$ & 8.9 & $7 \cdot 6,10 \cdot 5$ & $2 \cdot 6$ & $2 \cdot 0,3 \cdot 3$ & $1 \cdot 0$ & $0.7,1.4$ \\
\hline Use of firewood or carbon for cooking & $2 \cdot 0$ & $1 \cdot 6,2 \cdot 4$ & 5.4 & $4 \cdot 4,6 \cdot 7$ & $1 \cdot 0$ & $0.7,1.4$ & 0.2 & $0.1,0.4$ \\
\hline Public sanitary sewer system & $60 \cdot 2$ & $57 \cdot 3,63 \cdot 0$ & $19 \cdot 0$ & $16 \cdot 7,21 \cdot 5$ & 61.4 & $57 \cdot 9,64 \cdot 7$ & 88.5 & $86 \cdot 7,90 \cdot 1$ \\
\hline Public water network connected to household & $80 \cdot 8$ & $78 \cdot 4,83 \cdot 1$ & $45 \cdot 7$ & $41 \cdot 7,49 \cdot 7$ & $89 \cdot 1$ & $87 \cdot 1,90 \cdot 9$ & $99 \cdot 6$ & $99 \cdot 3,99 \cdot 8$ \\
\hline Electricity & 99.6 & $99.5,99.7$ & 98.9 & $98.5,99.2$ & 99.9 & $99.8,99.9$ & $100 \cdot 0$ & $99.8,99.9$ \\
\hline Motor vehicle (car, van, truck or motorcycle) & $16 \cdot 7$ & $15 \cdot 7,17 \cdot 7$ & 8.0 & $7 \cdot 0,9 \cdot 1$ & $14 \cdot 8$ & $13 \cdot 4,16 \cdot 4$ & $24 \cdot 2$ & $22 \cdot 5,26 \cdot 0$ \\
\hline Television (colour) & 89.9 & $89 \cdot 1,90 \cdot 6$ & 84.9 & $83 \cdot 2,86 \cdot 4$ & $91 \cdot 6$ & $90 \cdot 3,92 \cdot 7$ & $92 \cdot 2$ & $90 \cdot 9,93 \cdot 2$ \\
\hline Computer (laptop or desktop) & 34.4 & $32 \cdot 6,36 \cdot 3$ & 11.6 & $10 \cdot 3,13 \cdot 1$ & 33.5 & $31 \cdot 1,36 \cdot 1$ & $51 \cdot 2$ & $48 \cdot 9,53 \cdot 4$ \\
\hline Telephone & 40.9 & $38 \cdot 8,43 \cdot 0$ & $15 \cdot 3$ & $13 \cdot 4,17.5$ & 40.5 & $38 \cdot 0,43 \cdot 1$ & 59.2 & $56 \cdot 7,61 \cdot 6$ \\
\hline Cell phone & $91 \cdot 3$ & $90 \cdot 6,92 \cdot 0$ & $87 \cdot 1$ & $85 \cdot 5,88 \cdot 5$ & $92 \cdot 9$ & $91 \cdot 9,93 \cdot 9$ & 93.2 & $92 \cdot 2,94 \cdot 1$ \\
\hline
\end{tabular}

Education level is based on years of education and is based on mother's education level for children aged $<5$ years and adolescent women aged 11-19 years. 


\section{NS Public Health Nutrition}

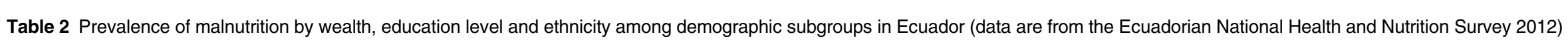

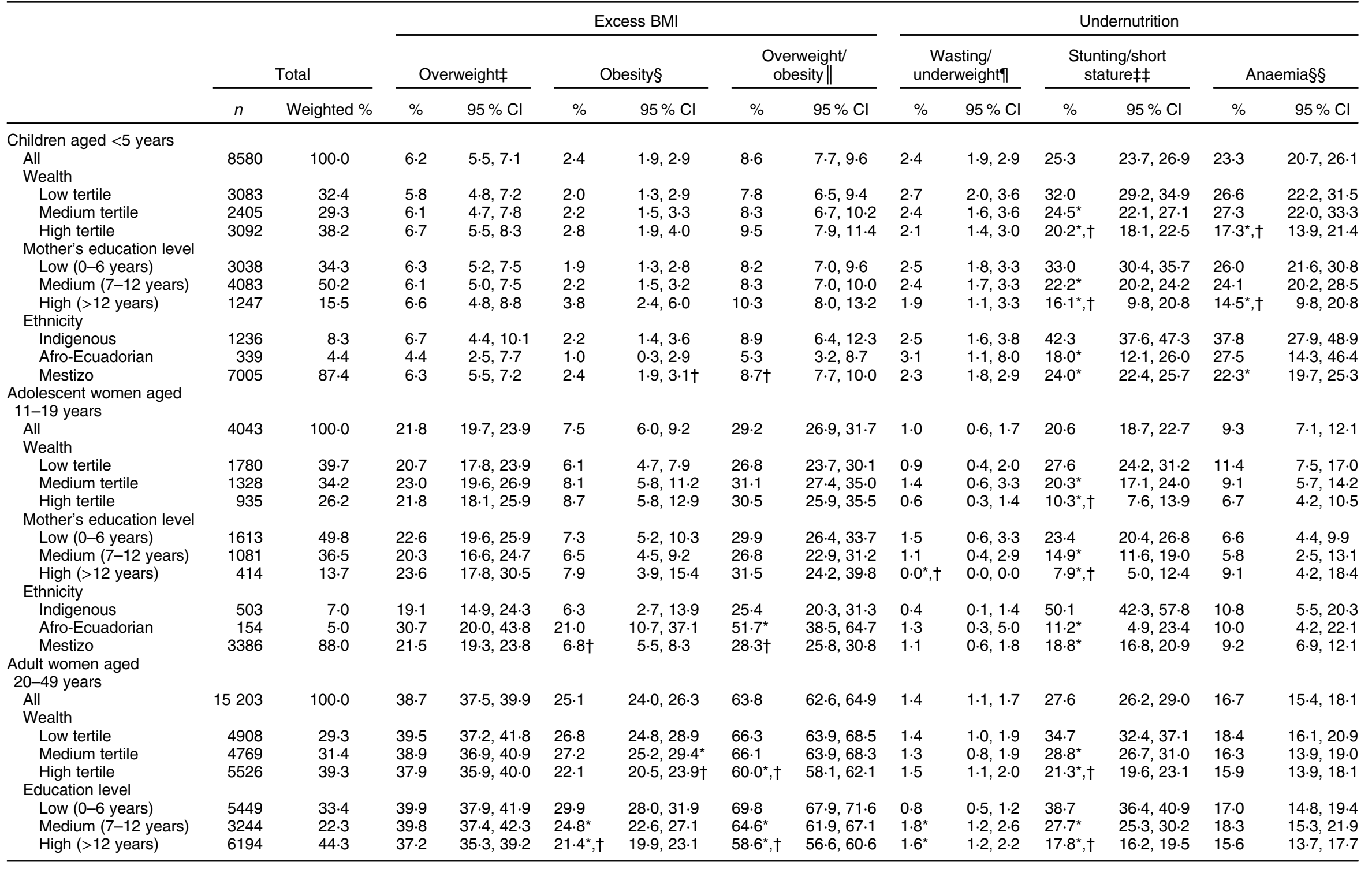



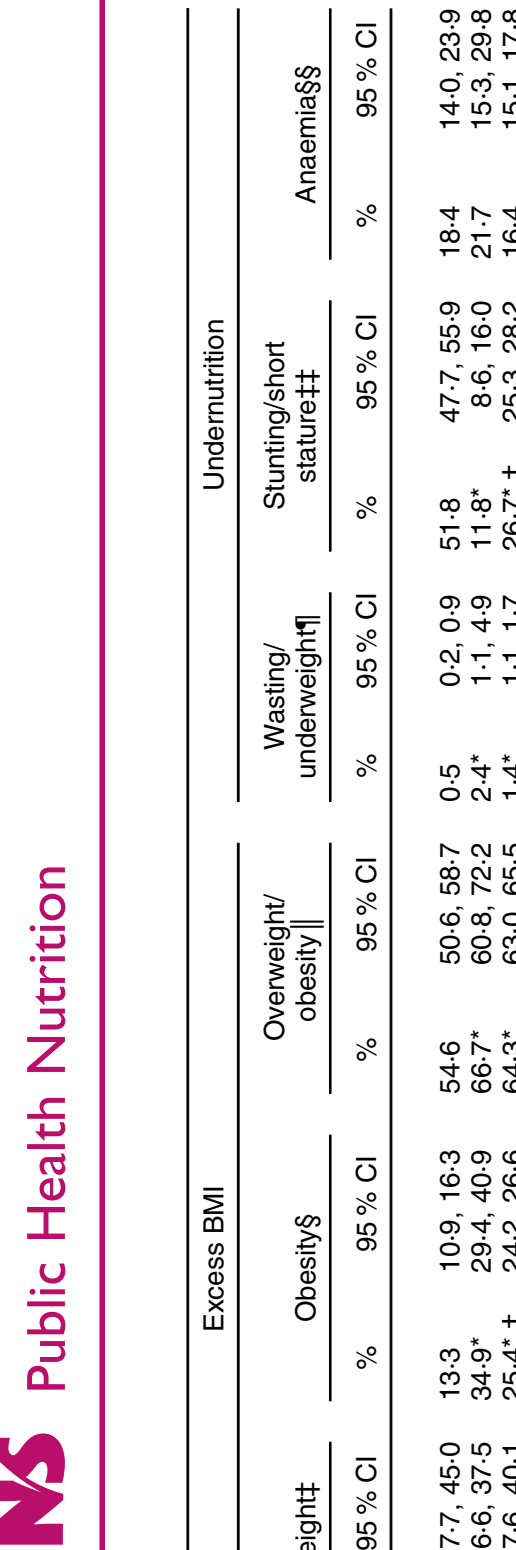

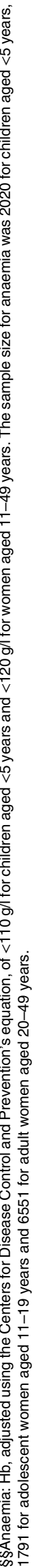

\section{Discussion}

In the present study we document important social inequalities regarding malnutrition indicators among children of pre-school age and women of reproductive age. Undernutrition in the form of stunting and anaemia disproportionately affects socially disadvantaged groups (low wealth, low education and indigenous). Differences in excess weight are smaller and vary by age group. For instance, among children aged $<5$ years the gaps are very small, whereas among adolescent and adult women, the most significant differences are observed in ethnic minorities (Afro-Ecuadorian) and the low education group.

Many factors help explain the overweight and obesity epidemic in the Ecuadorian context. On an individual level, excess weight results from an imbalance between energy consumed and energy expended. At the collective level, energy imbalances are related in part to improvements in socio-economic conditions, changes in occupational structure, rapid urbanization, and changes in the food supply and food environment. The food environment is characterized by an abundance of widely advertised, relatively inexpensive and highly palatable energy-dense foods. These trends, in turn, influence dietary preferences and have been accelerating in low- and middle-income countries like Ecuador ${ }^{(4,20)}$. The term 'obesogenic' is often used to describe a permissive environment that promotes food intake at levels well beyond the control of the individual resulting in excess body weight. Furthermore, physical activity levels have decreased as traditional lifestyles based on strenuous labour have changed dramatically in favour of more sedentary occupations and leisure activities ${ }^{(21-23)}$. Results from ENSANUT-ECU show that $21 \%$ of children and $26 \%$ of adolescents in urban areas spend $\geq 2 \mathrm{~h}$ on screen time per day, and this trend increases with socioeconomic status. Among the adult population in urban areas ( $>18$ years), $64 \%$ is sedentary and this proportion is higher in women $(74.4 \%)$ relative to men $(52.7 \%)^{(1)}$.

It has been documented that improvements in socioeconomic status in low- and middle-income countries like Ecuador initially increase rates of excess weight (particularly overweight) and decrease rates of undernutrition ${ }^{(24)}$. As rapid economic growth occurs, undernutrition remains high among the poor, whereas overweight develops initially among the wealthy. In the Ecuadorian context, we observe higher rates of overweight and obesity among children and women of low wealth groups, suggesting that the country is in a relatively advanced stage of the nutrition transition $^{(25)}$.

Various social, cultural and economic factors may be related to the distribution of malnutrition observed in Ecuador. During the past decade, significant economic and social transformations have occurred which can help contextualize these study findings ${ }^{(26)}$. Between 2006 and 2014 growth in gross domestic product in Ecuador averaged $4.3 \%$, which enabled increased social spending. 
(a)

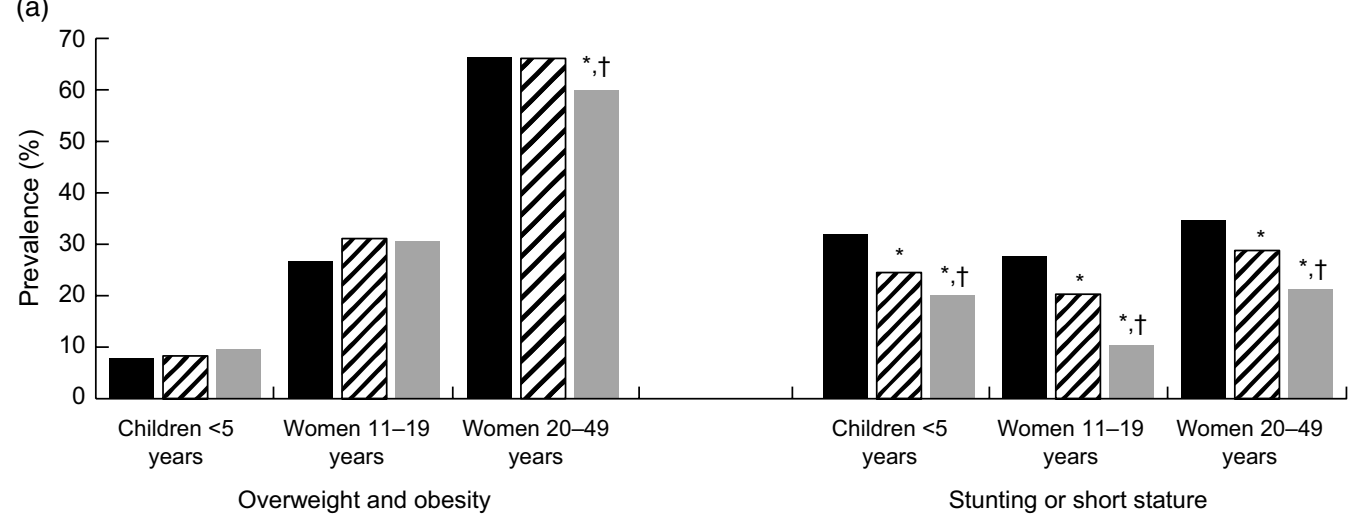

(b)

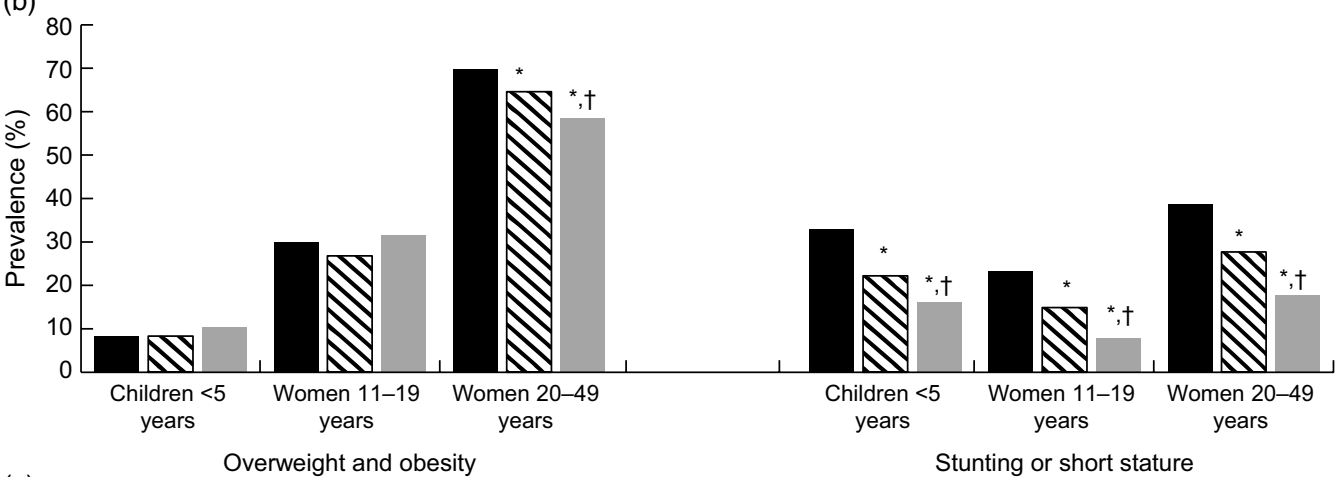

(c)

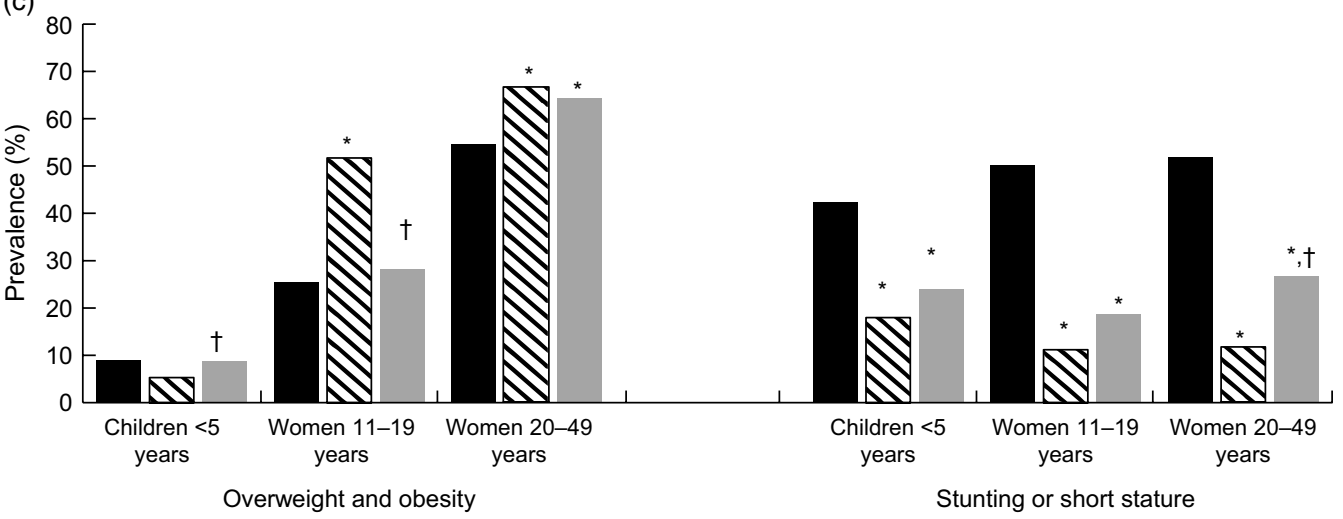

Fig. 1 Prevalence of overweight and obesity and of stunting or short stature by (a) wealth ( $\mathbf{\square}$, low tertile; $\mathbb{\mathbb { Q }}$, medium tertile; $\square$, high tertile), (b) education level ( $\mathbb{G}$, low; $\mathbb{\mathbb { N }}$, medium; , high) and (c) race or ethnicity ( $\mathbf{\square}$, indigenous; $\mathbb{\mathbb { N }}$, Afro-Ecuadorian;, mestizo) among children aged $<5$ years ( $n$ 8580), adolescent women aged $11-19$ years $(n$ 4043) and adult women aged 20-49 years ( $n 15203$ ) in Ecuador. Education level is based on years of education and is based on mother's education level for children aged $<5$ years and adolescent women aged $11-19$ years. ${ }^{*} P<0.05 \mathrm{v}$. low tertile/low education/indigenous; $\dagger P<0.05 v$. medium tertile/ medium education/Afro-Ecuadorian. (Data are from the Ecuadorian National Health and Nutrition Survey 2012)

During that period, poverty declined from 38.3 to $25 \cdot 8 \%$ and extreme poverty dropped from 12.9 to $5.7 \%$. The Gini coefficient decreased from 0.54 to 0.47 , reflective of greater income growth among the poorest segments of the population ${ }^{(27)}$.

Moreover, between 2007 and 2013 the country adopted education policies tripling the budget assigned to undergraduate public education, from \$US 1094.6 million in 2006 to \$US 2908.4 million in $2012^{(28)}$. A 10-year Education Plan, between 2006 and 2015, aimed to universalize early and primary education for children and increase upper secondary education enrolment to reach at least $75 \%$ of the population aged 16-18 years. These policies help explain the relatively high proportion of education observed among low wealth groups ${ }^{(28)}$.

In 2014 the Ecuadorian Government set a precedent by implementing mandatory front-of-pack labelling for regulating the sale of packaged foods and drinks. The traffic light labels display the levels of sugar, fats and salt with colour codes: red, yellow and green for high, medium and low content of these macronutrients, respectively. A qualitative evaluation of the initiative revealed that the traffic light labels helped raise consumers' awareness and understanding of the content of processed products ${ }^{(29)}$. 
The implementation of this initiative is an essential step for raising awareness among consumers. However, the traffic light food labelling by itself cannot influence a reduction in overweight and obesity. Rather, integrated policies of promotion and prevention must be implemented to address the problem of overweight and obesity in the context of the double burden of malnutrition.

The WHO has proposed a set of actions that have the potential to impact both sides of the double burden. These include the promotion of exclusive breast-feeding during the first 6 months, adequate early nutrition feeding practices (after 6 months), promotion of maternal nutrition, regulation of the food environment in schools and the implementation of marketing regulations ${ }^{(30)}$.

Furthermore, current interventions designed to address undernutrition must not inadvertently increase the risk of excess weight. For instance, initiatives to address micronutrient deficiencies through the fortification of staple foods that have the potential to produce further overweight and obesity should be discouraged.

Our study has some strengths and limitations. The strengths include the use of a nationally representative sample of Ecuadorian children of pre-school age and women of reproductive age. We included a comprehensive assessment of the main malnutrition problems affecting the Ecuadorian population, stratified by social indicators. Use of the MPI is an innovative approach to assess social inequalities. This index is aligned with the UN Sustainable Development Goals and allows to identify more precisely target groups for interventions and policy design. The main limitation of our study is the limited capacity to infer causality between socio-economic disparities and malnutrition indicators based on the cross-sectional nature of the data and the type of analysis conducted.

\section{Acknowledgements}

Acknowledgements: The authors would like to thank Emory University, Nutrition and Health Science Program, and Universidad San Francisco de Quito for supporting the authors with the time and resources to conduct this research, and the Latin American Nutrition Leadership Program (Programa LILANUT) for its coordination and support in the preparation of this manuscript. Financial support: M.J.R.-L. received PhD funding from the Laney Graduate School, Emory University and CONACYT Mexican Government. DSM Nutritional Products provided funds for the publication of this manuscript. DSM Nutritional Products had no role in the design, analysis or writing of this article. Conflict of interest: None of the authors declare a conflict of interest regarding this manuscript. Authorship: M.J.R.-L. conceived the research question and analysis plan, carried out the statistical analysis and wrote the manuscript. P.B. contributed to the statistical analysis. W.F.W. reviewed and contributed to writing the manuscript and interpreting the results. W.B.F. designed and supervised data collection and contributed to the development of the overall research and writing the manuscript. All of the authors read and approved the final manuscript. Ethics of human subject participation: This study was conducted according to the guidelines laid down in the Declaration of Helsinki and all procedures involving human subjects were approved by the Ethics Committee of Universidad San Francisco de Quito. Written informed consent was obtained from all subjects.

\section{References}

1. Freire WB, Ramírez-Luzuriaga MJ, Belmont $\mathrm{P}$ et al. (2013) Encuesta Nacional de Salud y Nutrición de la Población Ecuatoriana de Cero a 60 Años. ENSANUT-ECU 2011-2013. Tomo I (National Health and Nutrition Survey ENSANUTECU 2011-2013. Vol. 1). Quito: Ministerio de Salud Pública.

2. Freire WB, Dirren H, Mora JO et al. (1988) Diagnóstico de la Situación Alimentaria, Nutricional y de Salud de la Población Ecuatoriana Menor de Cinco Años: DANS (Food, Nutrition and Health Status Assessment of the Ecuadorian Population Less than Five Years: DANS). Quito: Consejo Nacional de Desarrollo, Ministerio de Salud Pública.

3. Freire WB, Silva-Jaramillo KM, Ramirez-Luzuriaga MJ et al. (2014) The double burden of undernutrition and excess body weight in Ecuador. Am J Clin Nutr 100, issue 6, 1636S-1643S.

4. Corvalán C, Garmendia M, Jones-Smith J et al. (2017) Nutrition status of children in Latin America. Obes Rev 18, $7-18$.

5. The World Bank (2014) World Development Indicators 2014. Washington, DC: World Bank Group.

6. Instituto Nacional de Estadística y Censos (2011) Censo de Población y Vivienda (Population and Housebold Census). Quito: INEC.

7. Malik K (2013) Human Development Report 2013. The Rise of the South: Human Progress in a Diverse World. New York: United Nations Development Programme.

8. Freire WB, Belmont P, Lopez-Cevallos DF et al. (2015) Ecuador's National Health and Nutrition Survey: objectives, design, and methods. Ann Epidemiol 25, 877-878.

9. de Onis M \& Habicht J-P (1996) Anthropometric reference data for international use: recommendations from a World Health Organization Expert Committee. Am J Clin Nutr 64 , 650-658.

10. Oshiro I, Takenaka T \& Maeda J (1982) New method for hemoglobin determination by using sodium lauryl sulfate (SLS). Clin Biochem 15, 83-88.

11. World Health Organization (2017) Guideline: Assessing and Managing Children at Primary Health-Care Facilities to Prevent Overweight and Obesity in the Context of the Double Burden of Malnutrition. Geneva: WHO.

12. de Onis M, Onyango AW, Borghi E et al. (2007) Development of a WHO growth reference for school-aged children and adolescents. Bull World Health Organ 85, 660-667.

13. Kozuki N, Katz J, Lee AC et al. (2015) Short maternal stature increases risk of small-for-gestational-age and preterm births in low-and middle-income countries: individual participant data meta-analysis and population attributable fraction. J Nutr 145, 2542-2550. 
Malnutrition inequalities in Ecuador

s67

14. World Health Organization (1995) The Use and Interpretation of Anthropometry. Report of a WHO Expert Committee. WHO Technical Report Series no. 854, pp. 312-409. Geneva: WHO.

15. World Health Organization (2011) Haemoglobin Concentrations for the Diagnosis of Anaemia and Assessment of Severity. Geneva: WHO.

16. Nested P (2002) Adjusting Hemoglobin Values in Program Surveys, pp. 2-4. Washington, DC: International Nutritional Anaemia Consultative Group, ILSI Human Nutrition Institute.

17. Alkire S, Conconi A \& Seth S (2014) Multidimensional Poverty Index 2014: Brief Methodological Note and Results. Oxford: Oxford Poverty and Human Development Initiative.

18. Alkire S \& Santos ME (2014) Measuring acute poverty in the developing world: robustness and scope of the multidimensional poverty index. World Lev 59, 251-274

19. Smedley A \& Smedley BD (2005) Race as biology is fiction, racism as a social problem is real: anthropological and historical perspectives on the social construction of race. Am Psychol 60, 16-26.

20. Popkin BM, Adair LS \& No SW (2012) Global nutrition transition and the pandemic of obesity in developing coontries. Nutr Rev 70, 3-21.

21. Delisle H, Ntandou-Bouzitou G, Ague V et al. (2012) Urbanisation, nutrition transition and cardiometabolic risk: the Benin study. Br J Nut 107, 1534-1544.
22. Mozaffarian D, Hoo T, Rem EB et al. (2011) Changes in diet and lifestyle and long-term weight gain in women and men. N Enol J Med 364, 2392-2404.

23. Waters WF (2001) Globalization, socioeconomic restructuring, and community health. J Community Health 26, 79-92.

24. Tzioumis E \& Adar LS (2014) Childhood dual burden of under- and overnutrition in low- and middle-income coontries: a critical review. Food Nut Bull 35, 230-243.

25. Popkin BM (1994) The nutrition transition in low-income countries: an emerging crisis. Nutr Rev 52, 285-298.

26. Larrea Maldonado C \& Camacho ZG (2013) Atlas de las Desigualdades Socioeconómicas del Ecuador. Quito: SENPLADES and Flacso.

27. The World Bank (2016) Reported de Pobreza for Consumo Ecuador 2006-2014. Washington, DC: World Bank Group.

28. Estarellas PC \& Bramwell D (2015) Ecuador, 2007-2014: attempting a radical educational transformation. Educ South Am 2007, 329.

29. Freire WB, Waters WF, Rivas-Mariño G et al. (2017) A qualitative study of consumer perceptions and use of traffic light food labelling in Ecuador. Public Health Nut 20, 805-813.

30. World Health Organization (2017) Double-Duty Actions for Nutrition: Policy Brief. Geneva: WHO.

https://doi.org/|0.1017/S1368980019002751 Published online by Cambridge University Press 